\title{
The quasi-optical analysis of Bessel beams in the far infrared
}

\author{
Neil Trappe ${ }^{\text {a,* }}$, Ronan Mahon a, William Lanigan a, J. Anthony Murphy a, \\ Stafford Withington ${ }^{b}$
}

a Experimental Physics, National University of Ireland, NUI Maynooth, Maynooth, Co Kildare, Ireland

b Cavendish Laboratory, Madingley Road, Cambridge CB3 OHE, UK

Received 2 December 2003

Available online 10 May 2004

\begin{abstract}
We discuss the Gaussian beam mode analysis of Bessel beams, eigen-solutions of the wave-equation in cylindrical polar coordinates which neither change form nor spread out as they propagate. Approximate, limited diffraction finite aperture, pseudo-Bessel beams having intense on-axis spots with large depths of field can be produced experimentally in the far infrared by using plastic conical lenses, known as axicons. We illustrate the physical insight provided by Gaussian beam mode analysis of such systems. Such pseudo-Bessel beams can be usefully approximated by high-order Gaussian-Laguerre modes, which have similar propagation characteristics. The size of the on-axis spot produced by an axicon, and its depth of focus, can be estimated from a single best-fit high-order Gaussian-Laguerre mode, and a more detailed description of behaviour can be achieved by adding a few additional modes of neighbouring orders. The strength of Gaussian beam mode analysis is that it is straightforward to model the propagation of Bessel beams through complex systems of long wavelength optical components, such as apertures, mirrors, and lenses. We report the experimental generation and measurement of a $0.1 \mathrm{THz}$ Bessel beam, and show that useful performance is possible for an axicon having a scale size just one order of magnitude greater than the wavelength. This work confirms the technical feasibility of designing and building long-wavelength optical systems based on Bessel beams.
\end{abstract}

(c) 2004 Published by Elsevier B.V.

Keywords: Quasi-optical analysis; Bessel beams; Axicons

\section{Introduction}

A cylindrically symmetric diffraction limited [1] free-space solution of the scalar wave equation

\footnotetext{
${ }^{*}$ Corresponding author. Tel.: +35317084658 ; fax: +353170 83313.

E-mail address: neal.a.trappe@may.ie (N. Trappe).
}

whose scale size is independent of propagation was first presented in 1987 by Durnin [1], and has the form [2]

$$
E(r, z, t)=J_{0}\left(k_{r} r\right) \exp \left(\mathrm{i}\left(k_{z} z-\omega t\right)\right),
$$

where the radial and axial wavenumbers, $k_{r}$ and $k_{z}$ respectively, are related to the free-space wavenumber, $k_{0}=2 \pi / \lambda_{0}=\omega / c$, by the dispersion 
relationship $k_{z}^{2}+k_{r}^{2}=k_{0}^{2}$. $\omega$ is the angular frequency, $\lambda_{0}$ the free-space wavelength, and $c$ the speed of light in vacuum. $J_{0}$ is the zero-order Bessel function of the first kind. For $0<k_{r} \leqslant k, k_{z}$ is real and the field profile, described by the zeroorder Bessel function $J_{0}\left(k_{r} r\right)$, possesses a sharp non-diverging central peak, surrounded by a set of concentric rings, all of which propagate, for an infinitely large beam, with constant amplitude and scale size. Although it is impossible to produce an ideal Bessel beam, because of its infinite lateral extent, a good approximation can nevertheless be realised in real physical systems.

At optical wavelengths, it is straightforward to produce "limited diffraction" [3] Bessel beams because components can be many thousands of wavelengths in diameter, but at submillimetre wavelengths the finite transverse extent becomes more of a problem. A key question is can Bessel beams having interesting and useful properties be generated at long wavelengths, and how can the behaviour of Bessel beams be modelled when they propagate through complex systems of finite-sized optical components? If suitable design procedures can be found then there will be numerous applications for this technology in areas as diverse as astronomical instrumentation and medical imaging.

Durnin et al. [2] originally used an annular aperture in the focal plane of a lens to produce an approximate Bessel beam, but optical configurations using axicons [4] proved to be more efficient. The use of computer generated holograms and axicons of finite diameter at longer millimetre wavelengths have also been reported; these produce 'pseudo' Bessel beams that maintain their form over a predetermined propagation distance before spreading rapidly beyond this range [5-7]. A basic configuration capable of producing Bessel beams in the far-infrared is shown in Fig. 1. When a Gaussian beam with a flat phase front (at a quasi-collimated waist position) is incident on the axicon, the additional focusing effect of the axicon causes strong near field interference effects in the region where the deflected beam overlaps with itself. Since, in the geometrical optics limit, the angles of overlap remain constant with the propagation distance, the profile of the interference pattern generated remains essentially invariant over the overlap region. The axial symmetry of the system results in the beam pattern having an amplitude profile that is approximated by a Bessel function of order zero $\left|J_{0}\left(k_{r} r\right)\right|^{2}$ [8]. Beyond the overlap region the on-axis beam amplitude falls rapidly to zero. In the far field of the axicon, the field has an annular form, as expected in the geometrical optics limit.

The propagation characteristics of a Bessel beam produced by an axicon are determined by the wavelength of the incident radiation, the angle of deviation $\beta$ of the axicon, and the effective radius $a$ of the axicon (i.e. the axicon rim for uniform illumination or weighted radius for an apodised profile such as an incident Gaussian beam). An estimate of the "limited diffraction depth of focus" $\Delta_{\mathrm{f}}$ of a pseudo-Bessel beam can be obtained from Snell's law, and is given by

$$
\Delta_{\mathrm{f}}=a / \tan \beta
$$

(a)

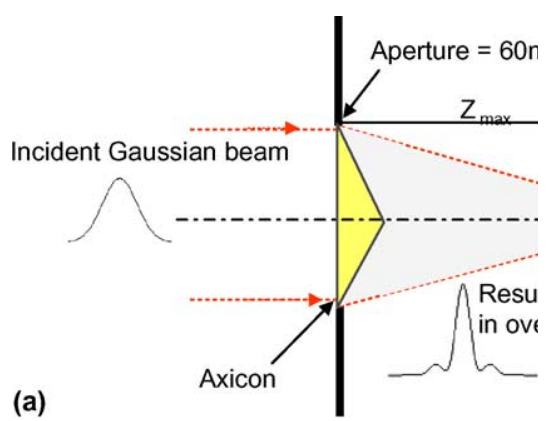

Fig. 1. (a) The geometrical configuration used to produce a Bessel beam. A Gaussian beam is incident on a conical lens, known as an axicon. (b) The cone angle $\gamma$, and the angle $\beta$ by which an incoming ray is refracted after passing through the axicon. 
where $\sin (\beta+\gamma)=n_{r} \sin \gamma$ : here, $n_{r}$ is the refractive index of the axicon, and $\gamma$ is the effective prism angle-see Fig. 1. This result is only an approximation, however, as a typical far-infrared axicon will only have a diameter that is one or two order of magnitudes greater than the wavelength and so edge diffraction effects will be more pronounced at far-infrared wavelengths than optical wavelengths, making the geometrical analysis less accurate.

The intense central spot of the beam produced by an axicon is very narrow, and furthermore appears to have an unphysically large depth of focus. In other words, if the central peak of a Bessel beam is approximated by a simple Gaussian having a radius of $W_{\mathrm{CM}}$, then the Bessel beam central peak remains collimated over distances much greater than $\pi W_{\mathrm{CM}}^{2} / \lambda$, the usual quasi-collimated confocal Rayleigh range [9]. Of course, the important physical difference between a simple Gaussian beam and a true Bessel beam is the presence of lowlevel diffraction rings in the Bessel beam. The apparent extension of the confocal range can be used in long-wavelength optical systems where a large depth of focus is needed in combination with high intensity and high spatial resolution [9].

An axicon having a finite diameter also produces an on-axis peak that diffracts very little over some focal range $\Delta_{\mathrm{f}}$. If the axicon is illuminated by a plane wave, however, diffraction effects can be very large [11]. These effects can be minimised by apodising the incoming beam such as, for example, by using Gaussian illumination [12]. For long wavelength systems, this approach is convenient, because highly tapered beams are generated by metallic waveguide and dielectric horns. In fact, the commonly used scalar horn has an aperture field that has the form of the central peak of the lowest order Bessel function $\left|J_{0}\left(k_{r} r\right)\right|^{2}$ [13].

Edge diffraction and truncation effects can be efficiently analysed and predicted using Gaussian beam mode analysis. A high-order GaussianLaguerre mode has an amplitude profile that is quite similar to a Bessel beam. They both possess a narrow central peak, extended diffraction rings, and remain collimated over a distance that is much larger than the confocal distance suggested by fitting a simple Gaussian to the central maximum. Of course for such high-order modes, only a small fraction of the total power is contained in the central peak, and it is the effective radius of the extended beam, including all of the diffraction rings, that determines the real Rayleigh range. By developing a multi-mode Gaussian-beam model, it should be possible to gain considerable insight into the behaviour of Bessel beams, and in particular, the way in which finite-sized axicons limit the performance that can be achieved: this topic is covered in Section 2. In addition, the analytical power of the Gaussian beam mode analysis can be brought to bear on the design and modelling of multi-element quasi-optical systems where axicons and Bessel beams are used. One can treat refocusing components, free-space propagation, vignetting, and aberration, in a straightforward and elegant manner, and feeds such as corrugated horns are readily described in terms of GaussianLaguerre mode coefficients. The analysis of standing waves caused by reflections is also well within the capability of the formalism. The propagation of Bessel beams through complex quasioptical systems is discussed in Section 3.

In Section 4, the experimental verification of a series of computer simulations is outlined. In particular, results obtained at $0.1 \mathrm{THz}$ with an axicon manufactured from high-density polyethylene are discussed. We show that it is possible to produce long-wavelength Bessel beams having central spots of just a few wavelengths in diameter. The combination of high intensity, resolving power, and depth of focus have many applications in optimising the performance of terahertz imaging systems. It is also possible to imagine developing novel axicon-coupled devices. For example, one could produce a compact Bessel-beam source by placing an axicon at the aperture of a large scalar horn. Axicons also provide alternative focussing devices to lenses for transporting and coupling power in beam guides. The analysis of such schemes can be realised in a straightforward manner by using Gaussian beam mode analysis.

\section{Gaussian beam mode analysis of axicon beams}

In this section, we present the basic Gaussian beam mode analysis (GBMA) of an axicon beam. 
In previous papers, we have shown the equivalence of the more typical Fresnel transform approach with GBMA in the case of classical diffraction problems and the diffraction theory of aberrations $[14,15]$. When modelling an axicon in the paraxial limit, the simplest approach is to consider the axicon as a thin phase-transforming screen of finite diameter at $z=0$, with the diffracted radiation suffering a conical phase transformation of $\delta(r) \propto r$. The total amplitude $E(X, Y, Z)$ at a point $(X, Y, Z)$ on an observation plane $z=Z$ can be calculated from the diffraction integral

$E(X, Y, Z) \propto \int_{0}^{2 \pi} \int_{0}^{a} \frac{\mathrm{e}^{-\mathrm{i} k_{0} \rho}}{\rho} \mathrm{e}^{-\mathrm{i} \delta(r)} r \mathrm{~d} r \mathrm{~d} \phi$,

where $\rho=\sqrt{(X-r \cos \phi)^{2}+(Y-r \sin \phi)^{2}+Z^{2}}, a$ is the radius of the axicon and $(r, \phi)$ are the polar co-ordinates of a point on the axicon plane (Fig. 2).

The presence of the conical axicon introduces a variation in the optical path length across the incident beam. The thickness of the cone at a radius $r$ is given by $t(r)=(a-r) \tan \gamma$, which leads to the phase transformation

$\delta(r)=\delta_{0}+k_{0}\left(n_{r}-1\right)(a-r) \tan \gamma$,

where $\delta_{0}$ is the phase lag through the centre of the axicon. Since the evolution of the beam is of interest, the diffraction integral (3) must be evaluated at a large number of points. This can be computationally intensive and also become numerically unstable as $z \rightarrow 0$, leading to the requirement of unreasonably large sampling rates. The modal approach does not does suffer from these shortcomings, and is, in general, more appropriate for the analysis of axicon beams in quasi-optical systems. The modal approach allows

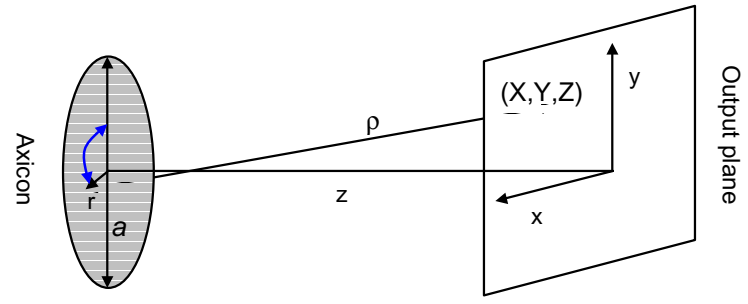

Fig. 2. The co-ordinate system used to describe Bessel beams using Fresnel diffraction techniques. one to analyse complex beam guide systems by building them up in a modular way, while at the same time keeping track of the evolution of the beam.

In Gaussian beam mode analysis [16], a paraxial monochromatic coherent beam, represented by a scalar electric field $E$, is expressed as a linear sum of independently propagating Gaussian modes $\Psi_{n}$. At any plane $z$, the field is of the form

$E_{0}(r, z)=\sum A_{n} \Psi_{n}(r, z, W(z), R(z))$,

assuming cylindrical symmetry. $W(z)$ and $R(z)$ are beam parameters that depend on the propagation distance $z$, and the $A_{n}$ are mode coefficients. Since axicon beams are axially symmetric, independent of azimuthal angle $\phi$, only the symmetric Laguerre-Gaussian modes of degree zero are required

$$
\begin{aligned}
\Psi_{n}(r, z)= & \frac{1}{W(z)} \sqrt{\frac{2}{\pi}} L_{n}\left(\frac{2 r^{2}}{W(z)^{2}}\right) \exp \left(-\frac{r^{2}}{W(z)^{2}}\right) \\
& \times \exp \left(-\mathrm{j} k\left(\frac{r^{2}}{2 R(z)}\right)\right) \times \exp (-\mathrm{j} k z) \\
& \times \exp \left(\mathrm{j}(2 n+1) \Delta \phi_{00}(z)\right) .
\end{aligned}
$$

The beam width parameter $W(z)$, phase front radius of curvature $R(z)$, and phase slippage $\Delta \phi_{00}(z)$ are functions of distance $z$ given respectively by

$W^{2}(z)=W_{0}^{2}\left[1+\left(\frac{z \lambda_{0}}{\pi W_{0}^{2}}\right)^{2}\right]$,

$R(z)=z\left[1+\left(\frac{\pi W_{0}^{2}}{\lambda_{0} z}\right)^{2}\right], \quad$ and

$\Delta \phi_{00}(z)=\tan ^{-1}\left(\frac{\lambda_{0} z}{\pi W_{0}^{2}}\right)$,

where $W_{0}$ is the minimum value of $W$, which occurs at $z=0$. If the field $E$ is known at some reference plane $z=z_{0}$, the mode coefficients can be calculated through overlap integrals of the general form

$$
A_{n}=\iint \Psi_{n}^{*}\left(r, z_{0}, W\left(z_{0}\right), R\left(z_{0}\right)\right) E_{0}\left(r, z_{0}\right) r \mathrm{~d} r \mathrm{~d} \phi .
$$


The modes $\Psi_{n}$ will propagate through a perfect beam-guide without scattering, and the output field at any plane can be reconstructed by adding together the modes as in [9]. For beams having a simple profile, the value of $W_{0}$ is usually chosen so as to maximise the power in the fundamental mode [9]. In this way, the fundamental mode can be used as a zeroth-order approximation of the intensity distribution, and $W$ gives the scale size of the beam on propagation. For a complicated beam, like a Bessel beam, however, the fundamental Gaussian mode is a very poor approximation, and a different choice of $W$ that is more physically meaningful is desirable. Ideally, we would like to find a single high-order Gaussian-Laguerre mode $\Psi_{n}(r, z W, R)$ (defined in terms of both its order $n$ and $W$ ) that is a reasonable approximation to the axicon beam. The physical behaviour of the Gaussian mode as it propagates then approximates the Bessel beam it represents.

When an axicon is introduced into a propagating beam, we can relate the input beam $E_{\text {in }}(r, \phi)$ to the output beam, using $E_{\text {out }}(r, \phi)=$ $E_{\text {in }}(r, \phi) \exp (\mathrm{i} \delta(r))$, with $\delta(r)$ given by Eq. (4). As in Eq. (3), this approach assumes that the axicon behaves like a thin lens, in the sense that the phase transformation occurs across a transverse plane at $z=0$, and diffraction effects within the thickness of the axicon are ignored. The mode coefficients of the axicon beam can then be evaluated

$$
\begin{aligned}
A_{n} & =\int_{0}^{r=a} \int_{0}^{\phi=2 \pi} \Psi_{n}^{*}\left\{E_{\text {out }}(r, \phi)\right\} r \mathrm{~d} r \mathrm{~d} \phi \\
& =\int_{0}^{r=a} \int_{0}^{\phi=2 \pi} \Psi_{n}^{*}\left\{E_{\text {in }}(r, \phi) \exp \left(\mathrm{i} k_{r} r-\mathrm{i} \delta_{0}^{\prime}\right)\right\} r \mathrm{~d} r \mathrm{~d} \phi,
\end{aligned}
$$

where $k_{r}=k_{0}\left(n_{r}-1\right) \tan \gamma$ and the constant $\delta_{0}^{\prime}=k_{0}\left(n_{r}-1\right) a \tan \gamma$.

Without loss of generality, we can assume that the input field is a simple Gaussian beam, and that the axicon is located at the waist position. Furthermore, corrugated conical horns produce Gaussian beams with typically $98 \%$ efficiency, and so such systems are easy to realise in practice [13]. The field at any plane beyond the axicon can be found, once the mode coefficients are known, by simply summing the modes according to
$E(r, z)=\Sigma A_{m} \Psi_{m}(r, z, W(z), R(z))$. The scale size of the beam evolves according to the beam width parameter $W(z)$, and the beam changes form as relative phase shifts accumulate between the different modes.

At this stage, we have still to determine what choice of beam width parameter at the axicon $W_{0}$ is best suited to producing a mode set that describes the Bessel beam efficiently. As described above, we ideally wish the Bessel beam to be approximated by either a single high-order Gaussian-Laguerre mode, or at least by a finite sum of a small number of neighbouring modes. This approach is analogous to the way in which the fundamental Gaussian mode can be chosen to approximate beams having simple tapered profiles. As an example, we take the case (closely related to the experimental arrangement described in Section 4) of a Gaussian beam having $W_{\text {in }}=45 \mathrm{~mm}$ incident on an axicon having radius $a=30 \mathrm{~mm}$, prism angle $\gamma=20^{\circ}$ and refractive index 1.52. For a range of values of $W_{0}$ between 5 and $25 \mathrm{~mm}$, we evaluate the corresponding mode coefficients. Fig. 3 below shows the fractional power carried by the first 12 modes for sample values of $W_{0}$. Choosing a value of $W_{0}=12 \mathrm{~mm}$ is the most efficient, as is clear from Fig. 3. The beam is then clearly well represented by a small number of modes bunched around $n=3$. The confocal distance of the mode set, $\pi W_{0}^{2} / \lambda=150 \mathrm{~mm}$, for $\lambda=3 \mathrm{~mm}$, is approximately equal to the predicted propagation distance for the Bessel beam, $\Delta_{\mathrm{f}}=170 \mathrm{~mm}$ (angle of deviation $\beta=11.1^{\circ}$ ). The most efficient mode set for describing the beam from a particular axicon, therefore, has a beam width parameter $W_{0}$ whose confocal distance matches the non-diverging distance given by(2). Combining the confocal distance with(2) gives

$$
W_{0} \approx \sqrt{\lambda a / \pi \tan \beta}
$$

giving $W_{0}=12.8 \mathrm{~mm}$ for the example being considered.

The best-fit high-order Gaussian-Laguerre mode possesses similar characteristics to the Bessel beam produced by the axicon. In fact, the similarity can be used as an alternative way of finding the best-fit beam-width parameter $W_{0}$ and the 


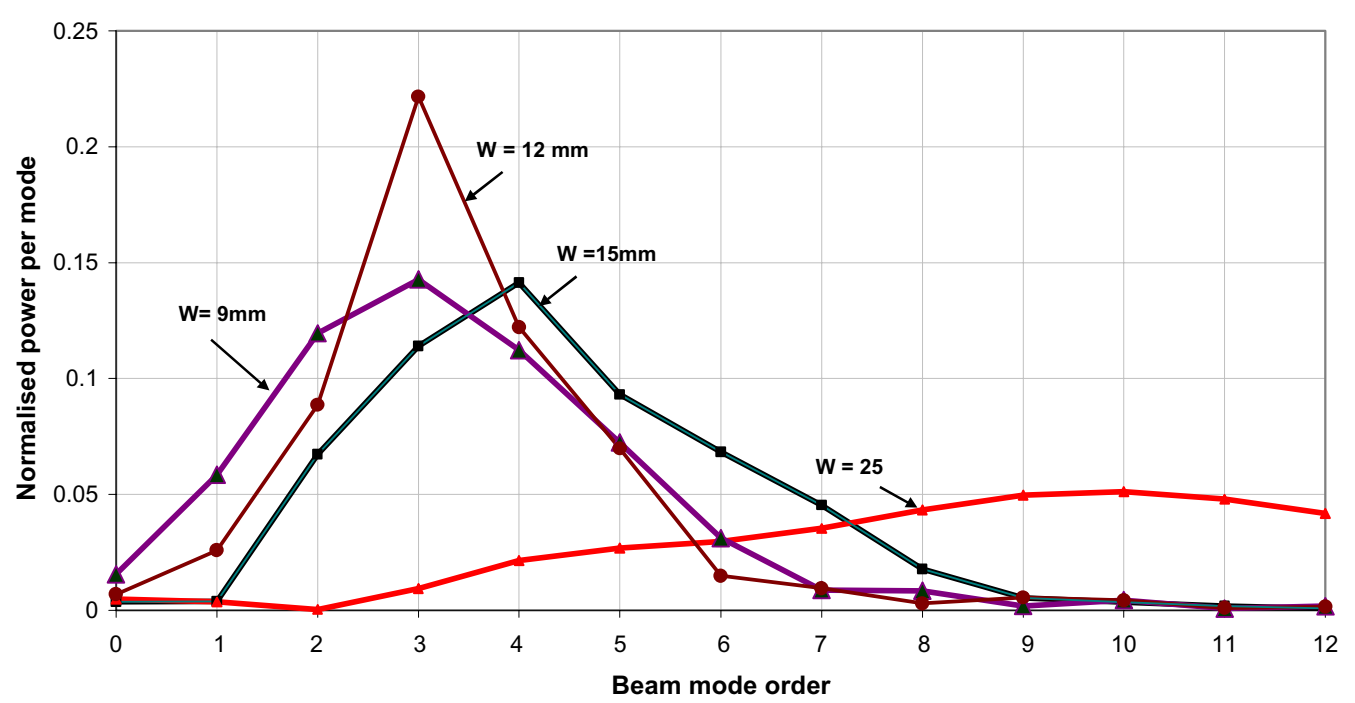

Fig. 3. Power distribution over the beam modes as a function of $W$. The most efficient $W$ is for $W=12 \mathrm{~mm}$.

associated order $n$. In other words, the axicon beam and Gaussian-Laguerre mode should have about the same width and have approximately the same number of in-phase zero crossings between the axis and the rim. For high-order GaussianLaguerre modes, the effective edge of the field (90\% power included) is given by $r=\sqrt{ }(2 n) W_{0}$, while the number of zero crossings is $n$. For an axicon beam, the number of zero crossings $n_{A}$ is given by the extra number of half wavelengths introduced into the optical path between the axicon axis and its rim, thus $n_{A}=2 a \tan \beta / \lambda$. Matching the widths of the Gaussian mode and axicon beam gives $\sqrt{ }(2 n) W_{0} \approx a$, and equating the number of zero crossings, $n \approx n_{A}=2 a \tan \beta / \lambda$, yielding $W_{0} \sqrt{ } a \lambda / 4 \tan \phi$. This value is consistent with the result given above, within the approximations assumed. Of course the exact mode, and value of $W_{0}$ that maximises the power in this mode, will depend on the nature of the beam that illuminates the axicon, and on the fact that the power is not perfectly uniformly distributed between the diffraction rings of the high-order Gaussian-Laguerre modes. Nevertheless, (10) is a useful approximation of the waist needed.

Furthermore, for the example considered above, where $a=30 \mathrm{~mm}$, we obtain $W_{0} \approx 12 \mathrm{~mm}$, yielding $n=3.13 \approx 3$, as expected. Table 1 shows
Table 1

The higher order mode coefficients of a Bessel beam using $W=12 \mathrm{~mm}$ for an axicon with radius $=30 \mathrm{~mm}$ and $\gamma=20^{\circ}$

\begin{tabular}{lll}
\hline Mode number & $\begin{array}{l}\text { Abs. value of coeff. } \\
\left|B_{m}\right|\end{array}$ & $\begin{array}{l}\text { Power content- } \\
\left|B_{m}\right|^{2}\end{array}$ \\
\hline 0 & 0.083067 & 0.0069 \\
1 & 0.160915 & 0.025894 \\
2 & 0.297835 & 0.088706 \\
3 & 0.47079 & 0.221643 \\
4 & 0.349583 & 0.122208 \\
5 & 0.264294 & 0.069851 \\
6 & 0.121817 & 0.014839 \\
7 & 0.097253 & 0.009458 \\
8 & 0.053844 & 0.002899 \\
9 & 0.074423 & 0.005539 \\
10 & 0.064989 & 0.004224 \\
11 & 0.032195 & 0.001037 \\
12 & 0.038399 & 0.001474 \\
13 & 0.030383 & 0.000923 \\
14 & 0.016992 & 0.000289 \\
15 & 0.033621 & 0.00113 \\
16 & 0.035108 & 0.001233 \\
17 & 0.019881 & 0.000395 \\
18 & 0.015174 & 0.00023 \\
19 & 0.023108 & 0.000534 \\
\hline
\end{tabular}

the fractional amounts of power present in the first 20 modes when $W_{0}=12 \mathrm{~mm}$, close to the optimum value. It can be seen that the maximum power is contained in the third order mode, and also sig- 


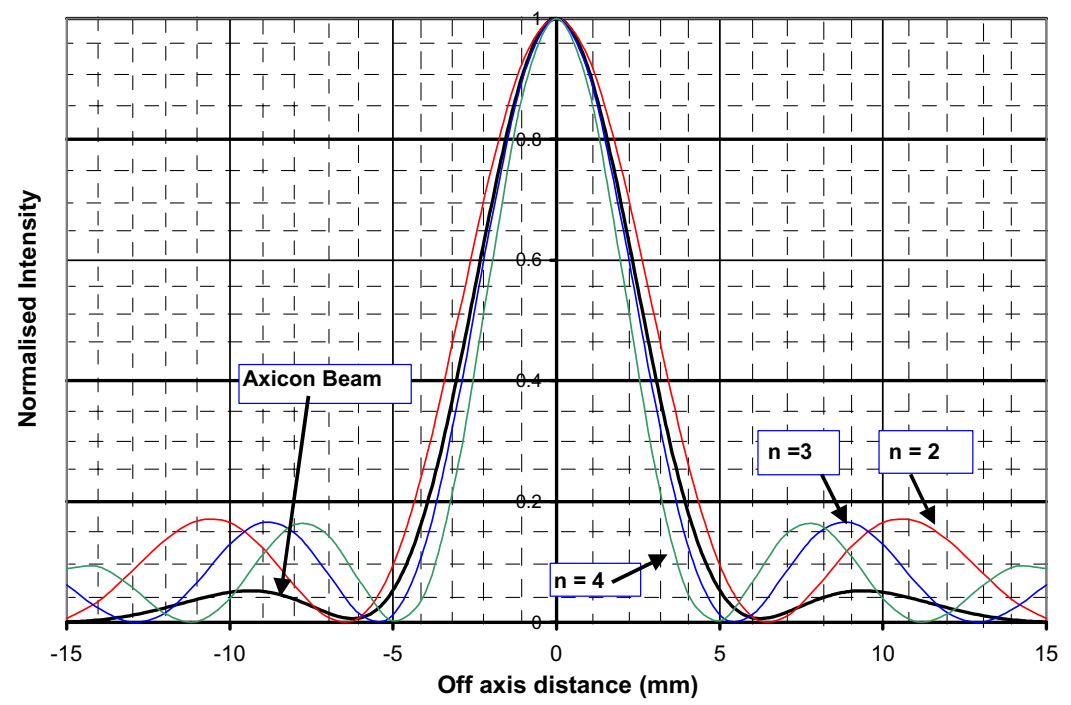

Fig. 4. Output Bessel beam compared to Laguerre-Gaussian mode of orders 3-5 (the modes containing most power.). The similar beam profiles indicate that the mode set used is a good description of the output field.

nificant power is contained in the neighbouring second, fourth and fifth modes. In Fig. 4, we plot the axicon beam at $z=80 \mathrm{~mm}$, (where the axicon beam best approximates a Bessel beam in terms of its intensity profile), together with the high-order Gaussian-Laguerre modes that contain most power. As can be seen, the mode profiles (normalised to unity on-axis) match the central peak of the Bessel beam quite well, indicating that this mode set is a natural fit to the Bessel beam. It is also noticeable, however, that the secondary maxima are slightly narrower than the Bessel beam. Nevertheless, when three modes are added together, the intensity of the central peak is enhanced, while the secondary maxima are smeared, and reduced in size, giving an excellent approximation to the axicon beam. Only after these modes have travelled through the confocal range does sufficient phase slippage accumulate such that the modes no longer add with the correct phases to form a Bessel-like profile.

The modes also begin to spread, causing power to be distributed over a larger area, reducing the on-axis intensity of the axicon beam. Fig. 5 is a density plot showing the evolution of the axicon beam amplitude with propagation away from the

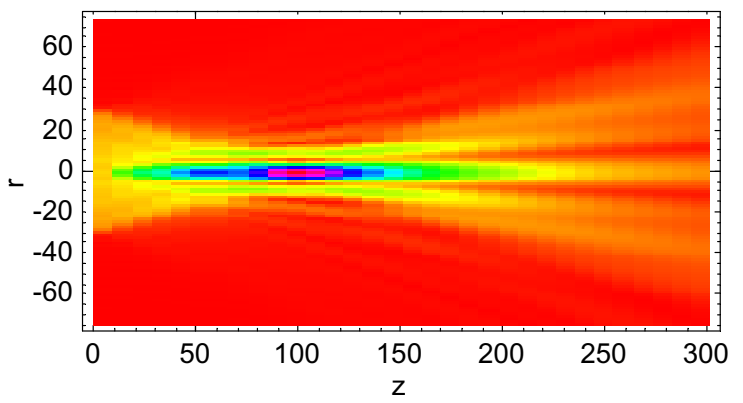

Fig. 5. Density plot showing the evolution of the axicon beam amplitude with propagation away from the "plane" of the axicon (modelled as a phase transformation at $z=0 \mathrm{~mm}$ ).

axicon reconstructed using the Gaussian beam mode technique. The axicon is at $z=0$.

Fig. 6 shows the amplitude and phase profiles for three representative values of $z: z=0,80 \mathrm{~mm}$ (approximately $50 \%$ of the focal range) and 300 $\mathrm{mm}$ (well beyond the focal range). Also plotted is the best-fit Gaussian-Laguerre mode of order 3. Both Figs. 5 and 6 describe how the beam from the axicon propagates.

At $z=0$, the amplitude has the same form as the truncated incident Gaussian, as it must, whereas by contrast, the phase has a large linear 

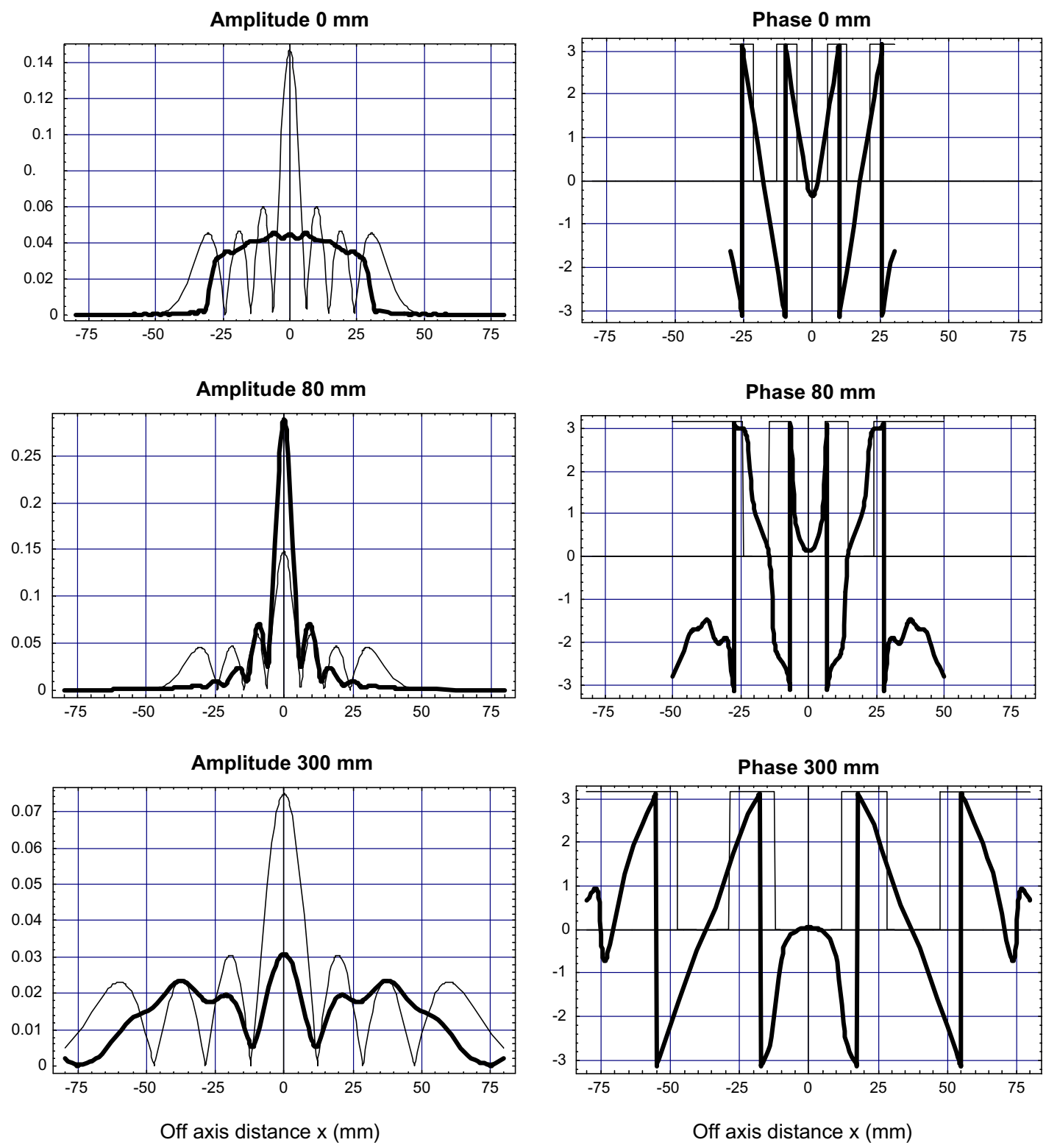

Fig. 6. Reconstructed beam patterns in amplitude and phase using GBMA (mode set with $W=12 \mathrm{~mm}$ ) at distances of 0,80 and 300 $\mathrm{mm}$ from the axicon. Also shown is the Laguerre mode with the highest coupling power to beam (faint line). The $x$-axis represents the off-axis distance $r$ in $\mathrm{mm}$ from the optical axis of the axicon. The $y$-axis represents amplitude (arbitrary units) or phase (in radians).

slope, which wraps around quickly through multiples of $2 \pi$ showing the presence of the phase transformation introduced by the axicon. On propagation, the amplitude pattern quickly evolves into a Bessel beam, because of the high spatial frequencies introduced by the phase transformation. The intensity profile soon develops an intense on-axis peak with faint diffraction rings (beyond $z=10 \mathrm{~mm}$ ). Also shown on Fig. 6 is the best-fit high-order Gaussian-Laguerre-Gaussian 
mode (faint line), which approximates the amplitude and phase of the Bessel beam. Beyond the limited diffraction-focal range, interference with the other higher-order modes eventually causes the Bessel beam pattern to disappear.

The on-axis intensity of the beam produced by the axicon does not remain constant in the overlap region (within $150 \mathrm{~mm}$ in this case: see Fig. 1), but varies with distance $z$ from the axicon. The intensity of a true Bessel beam of infinite extent would not vary. In the geometric limit, the axicon generates an on-axis intensity whose average value increases linearly with $z$ [8]. On the other hand, as already mentioned, in the case of long-wavelengths systems, diffraction effects caused by truncation at the edge of the axicon have an important influence on the on-axis intensity. Fig. 7a illustrates the variation in on-axis intensity, predicted using Gaussian beam mode analysis, for incident Gaussian beams having various widths relative to the size of the axicon. In generating Fig. 7, we have used an axicon radius of $30 \mathrm{~mm}$, as before. In modelling the system, all incident radiation outside of $30 \mathrm{~mm}$ was assumed to be terminated perfectly, similar of course to the experimental arrangement when absorbing material is used. The power in each incident Gaussian beam is normalised to unity, and so as the input Gaussian becomes large relative to the radius of the aperture, less power is coupled into the Bessel beam. This effect results in a lowering of the on-axis intensity for larger Gaussian beams, and also some diffraction effects (related to Poisson spots) are introduced. Fig. 7b shows the on-axis intensities of the Gaussian beams when the axicon is removed, but an aperture having the same radius of the axicon is left in place. It is evident that the on-axis intensity of the Bessel beam has features that are similar to the ordinary diffraction effects produced by an aperture: although, the Bessel beam has a much higher intensity peak, of course.

When designing a system, therefore, it is important to achieve the correct balance between making the incident Gaussian beam sufficiently big to get the greatest possible limited diffraction focal range, $\Delta_{\mathrm{f}}$, while at the same time, not making the beam so big that undesirable diffraction features are introduced. In Fig. 7a, it is also clear that if the waist is chosen correctly, the intensity of the onaxis spot builds up approximately linearly with distance, as in the geometrical case. The use of optical components with a range of focal lengths can be used to control the size of the incident Gaussian beam in an experimental configuration,
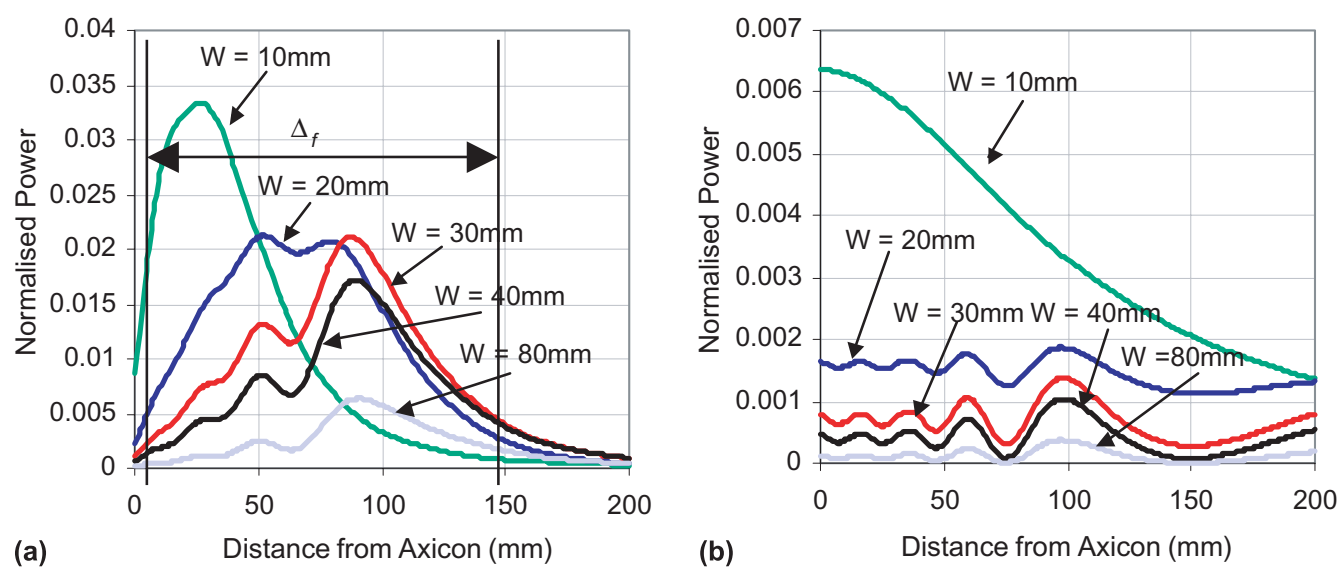

Fig. 7. (a) The variation of on-axis intensity of a Bessel beam produced by an axicon of radius $30 \mathrm{~mm}$ for Gaussian beams with a range of beam widths. (b) The variation of on-axis intensity of a range of simple Gaussian beams (no axicon) diffracted by an aperture with radius $30 \mathrm{~mm}$. 
and therefore we conclude that it is possible to design axicons that give reasonable performance at submillimetre wavelengths.

When the Bessel beam has propagated further than the limited diffraction-focal range, $\Delta_{\mathrm{f}}$, the central bright peak dissipates, and in the far field, the axicon beam possesses annular like structure with a possible faint on-axis spot.

\section{Axicon beams in complex quasi-optical systems}

Gaussian beam mode analysis is a very powerful tool for building up complicated quasi-optical systems involving multiple components (lenses, apertures, stops, curved off-axis mirrors etc.) [9,16-18]. Novel arrangements such as the coupling of two axicons facing each other can be readily investigated, and more complex optical fields that are partially coherent can be dealt with within the formalism [19]. Gaussian beam mode analysis also allows standing waves in multi-component systems to be modelled, which would be particularly beneficial if applied to Bessel beams [20].

According to this view, we consider the field incident on the axicon as already consisting of a modal sum having the form $E_{\text {in }}\left(r, z_{0}\right)=$ $\sum A_{n} \Psi_{n}\left(r, z_{0}, W, R\right)$. The effect of the axicon is to introduce a radial, linear phase delay: $\exp \left(\mathrm{i} k_{r} r\right)$, which affects each of the modes present in the summation. Thus, $E_{\text {out }}$ can be rewritten

$$
\begin{aligned}
E_{\text {out }}\left(r, z_{0}\right) & =\Sigma A_{n}\left\{\Psi_{n}\left(r, z_{0}, W, R\right) \exp \left(\mathrm{i} k_{r} r\right)\right\} \\
& =\Sigma B_{m}\left\{\Psi_{m}\left(r, z_{0}, W, R\right)\right\} .
\end{aligned}
$$

Clearly each phase-transformed modal field $\Psi_{n}^{\text {Axicon }}\left(r, z_{0}, W, R\right)\left(=\left\{\Psi_{n}\left(r, z_{0}, W, R\right) \exp \left(\mathrm{i} k_{r} r\right)\right\}\right)$ is no longer a free-space mode, but has to be itself represented as a sum of true modes $\Psi_{n}^{\text {Axicon }}\left(r, z_{0}, W, R\right)=\Sigma S_{m n} \Psi_{m}\left(r, z_{0}, W, R\right) . S_{m n}$ can then be thought of as the elements of a scattering matrix that represents the axicon. The elements are therefore given by

$$
S_{m n}^{\text {Axicon }}=\int \Psi_{m}^{*}(r, \phi)\left\{\Psi_{n}(r, \phi) \exp \left[\mathrm{i} k_{r} r\right]\right\} r \mathrm{~d} r \mathrm{~d} \phi .
$$

The output mode coefficients $B_{m}$ are now given by the alternative relationship $B_{m}=\Sigma S_{m n} A_{n}$. Thus, in mode amplitude space the set of input mode coefficients $A_{n}$ have been transformed into the set of output mode coefficients $B_{m}$ by the axicon represented by the scattering matrix $S_{m n}$.

The scattering matrix approach can be computationally intensive as a much greater number of integrations are required than the simpler approach taken in Section 2. However, the scattering matrix $S_{m n}$ is input-field independent, and can thus be used to describe the effect of the axicon on any general incoming field distribution. With this approach, it is also easy to model the effect of an aperture or other optical component placed in the path of a Bessel beam produced by an axicon [18]. In this case, we simply need the scattering matrix of the component, such that the propagating mode coefficients can be modified accordingly. However, it is also important to keep track of the component mode phase slippages in this scheme.

As an example, we take the system described in Section 2. Now, however, we place an aperture at a distance of $50 \mathrm{~mm}$ from the axicon (about $35 \%$ of the limited diffraction range in front of the axicon). The scattering matrix for an aperture is determined in a similar way to that of the axicon. The truncated incident field at the aperture can be written as the incident sum of truncated modes which must then be re-expressed as a new sum of true untruncated modes (in a similar way to the case of the axicon) in order to model propagation beyond the aperture. Thus,

$$
\begin{aligned}
E_{\text {out }}\left(r, z_{0}\right) & =\Sigma A_{n}\left\{\Psi_{n}\left(r, z_{0} ; W, R\right)\right\}^{\mathrm{T}} \\
& =\Sigma B_{m}\left\{\Psi_{m}\left(r, z_{0} ; W, R\right)\right\},
\end{aligned}
$$

where the superscript $T$ represents modal truncation. In this case the elements of the scattering matrix relating the $B_{m}$ to the $A_{n}$ through $B_{m}=\Sigma S_{m n} A_{n}$ are again given by overlap modal integrals, in this case of the form:

$$
\begin{aligned}
S_{m n}^{\text {Aperture }} & =\int \Psi_{m}^{*}(r, \phi)\left\{\Psi_{n}(r, \phi)\right\}^{\mathrm{T}} r \mathrm{~d} r \mathrm{~d} \phi \\
& =\int_{\text {Aperture }} \Psi_{m}^{*}(r, \phi)\left\{\Psi_{n}(r, \phi)\right\} r \mathrm{~d} r \mathrm{~d} \phi
\end{aligned}
$$


Clearly, the $S_{m n}$ values are obtained by evaluating the integral over the physical area of the aperture, as in [18]. Between the optical components the Gaussian modes accumulate mode dependent relative phase slippages, which can be incorporated into the scattering matrix formalism by representing this propagation in terms of a diagonal scattering matrix where the diagonal elements contain the phase slippage elements, $P_{n m}=\delta_{m n} \exp \left(\mathrm{j} \phi_{m}\right)$. The phase slippage terms are then associated with the mode coefficients rather than included in the functional form of the modal fields. If one can ignore standing waves then the overall scattering matrix for the axicon-aperture system is given by the matrix product of the three matrices $[\mathbf{S}]^{\text {Total }}=[\mathbf{S}]^{\text {Aperture }} \cdot[\mathbf{P}] \cdot[\mathbf{S}]^{\text {Axicon }}$.

The total scattering matrix of axicon-aperture system is thus readily evaluated, and the vector of mode coefficients is evaluated for the region beyond the aperture by multiplying the vector of incident mode coefficients at the axicon by this matrix. The beam pattern in the region beyond the aperture can then be reconstructed in a straightforward manner. Fig. 8 illustrates the effect of thus placing an aperture in the beam of Fig. 5: the two plots correspond to different sized apertures. As can be seen, stopping the beam down to the radius of the central spot (6 $\mathrm{mm}$ approximately) causes the beam to diffract severely, destroying the large depth of focus of the Bessel beam of Fig. 5. This effect is expected on physical grounds, because the higher order modes are preferentially attenuated thus removing their narrow intense central peaks from the beam. An alternative interpretation, is that the rings of the Bessel beam are removed, thus leaving a single narrow peak, with a reduced depth of focus. After the aperture, the beam spreads as though it were a simple Gaussian having a width equal to the central maximum (about $2 \lambda$ in this case). Thus, in general, the unique features of a Bessel beam can be severely compromised by the presence of any vignetting of the field. Also shown is the case where the truncation level is less severe so that the first ring of the diffraction pattern is allowed through also. The beam recovers some of its depth of field as expected.

It is even possible to include the effects of standing waves within this formalism provided the reflections from the axicon surfaces are adequately modelled. This follows a very similar analysis to that for lenses and dielectric windows discussed in [20]. The determination of the overall scattering matrix in that case is more complicated as the reflections are taken into account.

\section{Experimental set-up and results}

To validate the model, an experiment was undertaken to measure the field produced by an axicon at $100 \mathrm{GHz}$ (see Fig. 9). The axicon was manufactured from high density Polyethylene with a diameter of $60 \mathrm{~mm}$ and a prism angle of $20^{\circ}$ (cone apex angle of $140^{\circ}$ ). The refractive index of high density polyethylene at $100 \mathrm{GHz}$ is $n_{r}=1.52$ [9], giving an angle of deviation $\beta$ of $11.1^{\circ}$. Using(2), a limited diffraction range of $150 \mathrm{~mm}$ is calculated geometrically. The radius $r_{1}$ of the
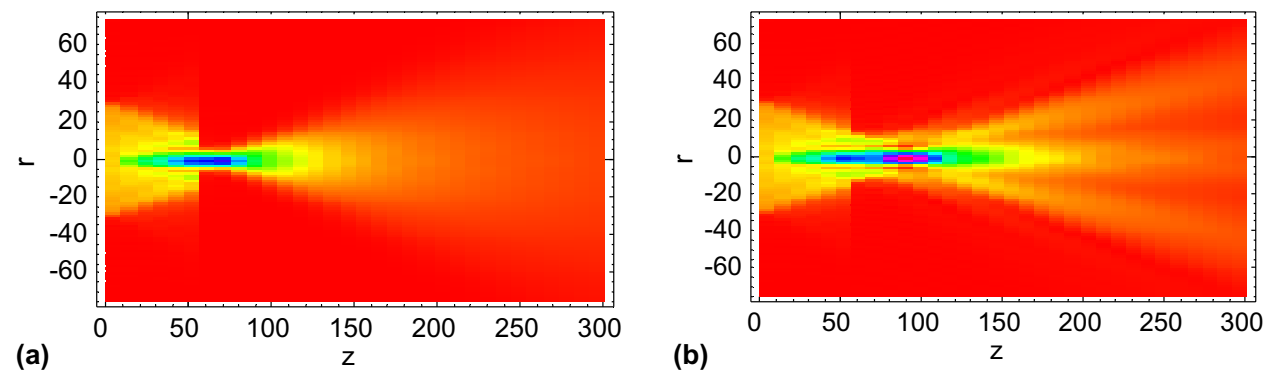

Fig. 8. Effect of truncation at an aperture stop for truncation radii of $r=6 \mathrm{~mm}$ and $12 \mathrm{~mm}$ (see also Fig. 5). 


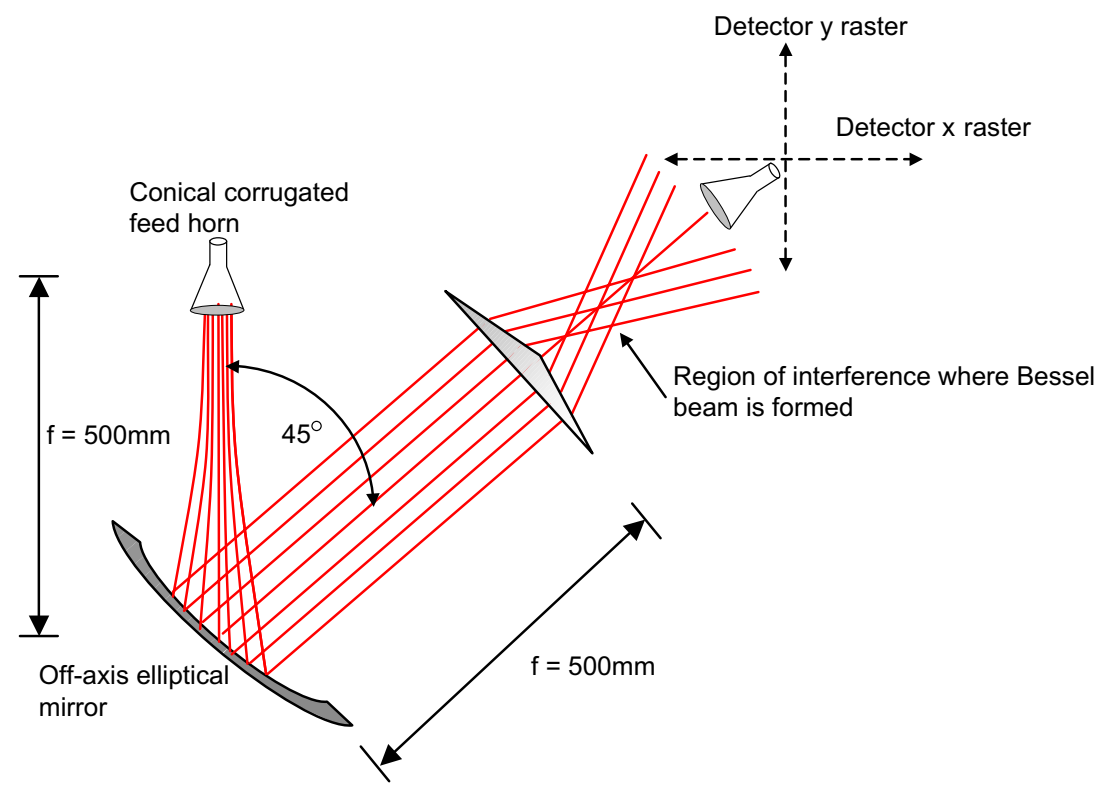

Fig. 9. Experimental set-up to investigate the output field from the axicon. A $x-y$ scanner is used to record the output field in a rectangular area.

central peak of the Bessel beam produced by the axicon can be determined roughly from the first zero of the Bessel function

$J_{0}\left(k r_{1} \sin \beta\right)=0$,

and given by [10]

$r_{1}=2.4048 \lambda / 2 \pi \sin \beta$.

For the particular axicon used in the experiment, a beam with a radius of $5.8 \mathrm{~mm}$ is predicted. The confocal range $\pi W^{2} / \lambda$ of an equivalent Gaussian with a beam radius $W$ of $5.8 \mathrm{~mm}$ is calculated to be $35.2 \mathrm{~mm}$ at $100 \mathrm{GHz}$, compared to $150 \mathrm{~mm}$ for the Bessel beam. This illustrates the large depth of focus that is available with a spot size of just a few wavelengths. Increasing the prism angle of the axicon can reduce the size of the central maximum. Narrow Bessel beams with a radius of $3 \mathrm{~mm}$ (order of a wavelength) can be produced with an axicon prism angle of $33^{\circ}$. In this case, however, the beam remains invariant over a shorter distance $\Delta_{\mathrm{f}}=71 \mathrm{~mm}$.

To measure the beam, a Gunn diode with an operating frequency range of $95-105 \mathrm{GHz}$ was used. The operating frequency set to $100 \mathrm{GHz}$ and a profiled corrugated horn (aperture radius 10.5 $\mathrm{mm}$, axial length $105 \mathrm{~mm}$ ) was used as the radiating antenna. The measured and predicted farfield patterns of the horn show low sidelobes and hence good Gaussicity. The phase centre (waist position) of the corrugated horn (with $W_{0 \mathrm{~h}}=6.1$ $\mathrm{mm}$ ) lies a distance of $18.6 \mathrm{~mm}$ behind the aperture and coincides with the focal point of an off-axis parabolic mirror $(f=500 \mathrm{~mm})$, which collimates the beam incident on the axicon. The angle of throw of parabolic mirrors was $45^{\circ}$. In this optical configuration, a corresponding Gaussian waist of radius $W$ of $78 \mathrm{~mm}$ is produced at the axicon with a flat phase front. The apodisation is thus relatively slight. A Schottky-diode detector was mounted on a computer-controlled XY scanner, at the output plane. The detector feed was a flared wave-guide of aperture diameter $2 \mathrm{~mm}$. Data was collected from the defined area and read to a PC via a variable gain scaling amplifier and a 16-bit $\mathrm{A} /$ D (analogue-digital) converter. A two-dimensional intensity pattern was thus measured with a dynamic range of $36 \mathrm{~dB}$. The scanner was capable of covering an area of $550 \mathrm{~mm} \times 550 \mathrm{~mm}$ with a step resolution of $0.3 \mathrm{~mm}$. Microwave absorbing 

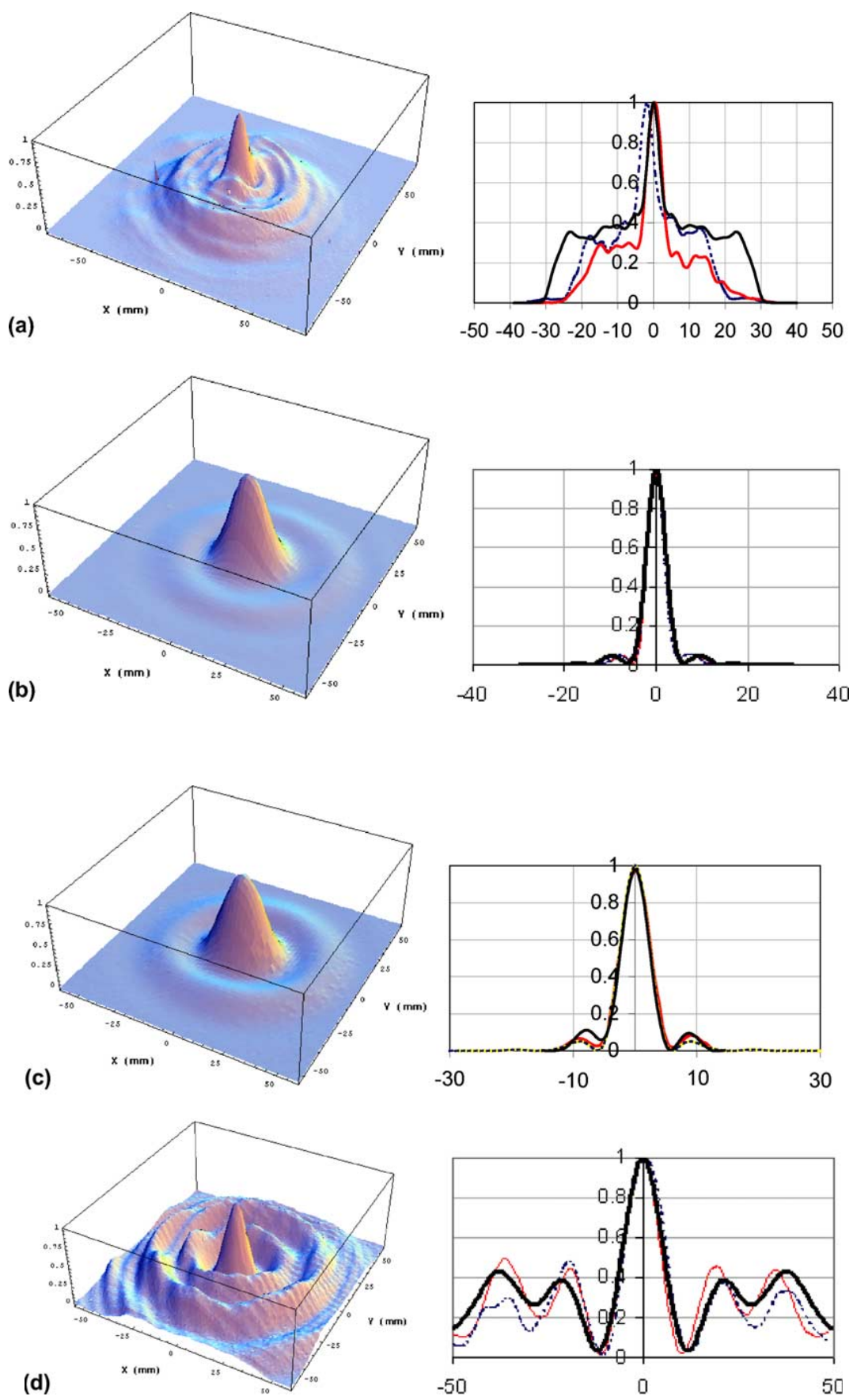

Fig. 10. Comparison experimental results with GMBA predictions. (a) Beam pattern $5 \mathrm{~mm}$ from axicon-rings beginning to form but incident Gaussian illumination still visible (heavy line $=$ theory, thin line $=x$ cut, dashed line $=y$ cut). (b) Beam pattern at $50 \mathrm{~mm}$ from axicon-intense central spot has now formed (heavy line $=$ theory, thin line $=x$ cut, dashed line $=y$ cut). (c) Beam pattern $100 \mathrm{~mm}$ from axicon-intense central spot close to centre of limited diffraction (heavy line $=$ theory, thin line $=x$ cut, dashed line $=y$ cut). (d) Beam pattern $300 \mathrm{~mm}$ from axicon - beyond limited diffraction focal range. Central intense spot begins to dissipate and has same intensity as rings (heavy line $=$ theory, thin line $=x$ cut, dashed line $=y$ cut). 
material was used to avoid unwanted reflections from the component mountings.

The experimental data is compared with theoretical results, calculated using Gaussian beam mode analysis, in Fig. 10. A cylindrically symmetric Bessel beam was indeed produced, with an intense on-axis spot and large depth of focus as predicted. Good agreement was achieved between simulation and experiment for values of $z>10$ $\mathrm{mm}$, verifying the theory and the practical realisation of a Bessel beam at millimetre wavelengths. At the 5-mm position (at the axicon), the Bessel beam profile was not fully formed. The beam from the axicon, however, was slightly narrower than the simulations predict. This disagreement is likely to be due to the approximation that the axicon behaves as a thin phase-transforming surface. Nevertheless, Gaussian beam mode analysis manages to predict, reasonably well, even the way in which the Bessel beam forms.

It is also interesting to point out that at distances greater than $150 \mathrm{~mm}$ the intensity of the Bessel beam fell off quickly. The on-axis intensity did not remain constant with propagation distance, but varied with distance as expected.

\section{Conclusions}

In this paper, we have illustrated how Gaussian beam mode analysis can be used to analyse the long wavelength behaviour of systems incorporating axicons. Gaussian beam mode analysis is computationally more efficient than the traditional Fresnel diffraction techniques, and also offers the advantage of giving physical insight into the way in which Bessel beams form and propagate. A Bessel beam can be approximated by a finite sum of a few neighbouring high-order Gaussian-Laguerre modes. The main advantage of the Gaussian mode approach is that it is straightforward to study the consequences of using a finite size axicon, and what happens when a Bessel beam propagates through a complicated sequence of optical components. In the paper, we illustrated the technique by propagating a Bessel beam through an aperture. We also reported the experimental generation of a Bessel beam at $0.1 \mathrm{THz}$; it was clear the theory predicted the true experimental behaviour exceptionally well. Our work opens up the possibility of designing and using Bessel beams at far-infrared and $\mathrm{THz}$ frequencies.

\section{Acknowledgements}

The authors wish to acknowledge the financial support of Science Foundation Ireland and the Irish Research Council for Science Engineering and Technology.

\section{References}

[1] J. Durnin, Exact solutions for non-diffracting beams. I. The scalar theory, J. Opt. Soc. Am. 4 (1987) 651-654.

[2] J. Durnin, J.J. Miceli Jr., J.H. Eberly, Diffraction free Bessel beams, Phy. Rev. Lett. 58 (1987) 1499-1501, pp. 651-654, 1987.

[3] J.-Y. Lu, Designing limited diffraction beams, IEEE Trans. Ultrason. Ferroelect. Freq. Control 44 (1) (1997) 181-193.

[4] G. Indebetouw, Nondiffracting optical fields: some remarks on their analysis and synthesis, J. Opt. Soc. Am. A 6 (Jan) (1989) 150-153.

[5] J. Turunen, A. Vasara, Friberg, Holographic generation of diffraction-free beams, Appl. Opt. 27 (1988) 3959-3962.

[6] J. Salo et al., Holograms for shaping radio-wave fields, J. Opt. A: Pure Appl. Opt. 4 (2002) 161-167.

[7] S. Monk, J. Arlt, D.A. Robertson, J. Courtial, M.J. Padgett, The generation of Bessel beams at millimetrewave frequencies by use of an axicon, Opt. Commun. 170 (1999) 213-215.

[8] I.C. Laycock, S.C. Webster, Bessel beams: their general and application, GEC J. Res. 10 (1) (1990).

[9] P.F. Goldsmith, Quasi-optical Systems: Gaussian Beam Quasi-optical Propagation and Applications, IEEE Press, 1998.

[10] Z. Ding, H. Ren, Y. Zhao, J. Stuart Nelson, Z. Chen, High resolution optical coherence tomography over a large depth range with an axicon lens, Opt. Soc. Am. Opt. Lett. 27 (4) (2002).

[11] Z. Jiang, Q. Lu, Z. Liu, Propagation of apertured Bessel beams, Appl. Opt.-OT 34 (31) (1995) 7183.

[12] J. Arlt, K. Dholakia, L. Allen, M.J. Pagett, Efficiency of second harmonic generation with Bessel beams, Phys. Rev. A 60 (3) (1999) 2438-2441.

[13] R.J. Wylde, Millimetre wave Gaussian beam mode optics and corrugated feed horns, IEEE Proc. 131 (1984) 158-262.

[14] J.A. Murphy, A. Egan, Examples of Fresnel diffraction using Gaussian modes, Eur. J. Phys. 14 (1993) 121-127.

[15] N. Trappe, J.A. Murphy, S. Withington, The Gaussian beam mode analysis of classical phase aberrations in 
diffraction-limited optical systems, Eur. J. Phys. 24 (2003) 403-412.

[16] D. Martin, J. Bowen, Long wave optics, IEE Trans. Micro. Theory Technol. 41 (10) (1993) 1676-1689.

[17] S. Withington, J.A. Murphy, K.G. Isaak, On the representation of mirrors in beam waveguides as inclined phasetransforming surfaces, Infrared Phys. Technol. 36 (1995) $723-734$.

[18] J.A. Murphy, S. Withington, A. Egan, Mode conversion at diffracting apertures in millimetre and submillimeterwave optical systems, IEEE Trans. Microwave Theory Tech. 41 (1993) 1700-1702.

[19] S. Withington, J.A. Murphy, Modal analysis of partially coherent submillimeter-wave quasi-optical systems, IEEE Trans. Antennas Propagation 46 (1998) 16511659.

[20] J.A. Murphy, N. Trappe, S. Withington, Gaussian beam mode analysis of partial reflections in simple quasi-optical systems fed by horn antennas, Infrared Phys. Technol. 44 (2003) 289-297. 